\title{
Filmpädagogik
}

\author{
Zurück zur Übersicht über den \\ Themenschwerpunkt
}

\section{Filmmusik im Kontext von Film- und Medienpädagogik}

\section{PETER IMORT}

\section{Einführung}

Filmmusik scheint eher am Rande der medienpädagogischen Beschäftigung mit Film zu liegen. Nur wenige Filme werden in erster Linie wegen der Musik angesehen, dennoch ist ihre Bedeutung wesentlich und untrennbar mit filmischen Produktionen verbunden. Die Reihe bedeutender Filme/Filmmusiken ist endlos lang, nicht erst seit der auf Schallplatte separat veröffentlichten und kommerziell äußerst erfolgreichen Filmmusik zum „Dschungelbuch“ (USA 1942; R: W. Reitherman; M: M. Rózsa), deren erste Schallplattenauflage die damals immense Höhe von 14.000 verkauften Exemplaren erreichte und noch heute zu den Ohrwürmern in den Kinderzimmern gehört.

Gegenwärtig gehört die Praxis separater (Kino-)Filmmusik-Veröffentlichungen zur gängigen PR-Strategie im Zuge eines umfassenden Merchandisings. Nicht immer überlebt die Musik diese Trennung von Bild und Ton so erfolgreich wie in der aktuellen Produktion „Les Choristes“ (dt. „Die Kinder des Monsieur Mathieu“; F 2004; R: C. Barratier; M: B. Coulais/C. Barratier) oder natürlich in Musikfilmen wie „Rhythm is it“ (R: T. Grube; M: I. Strawinsky/K. S. Elias) oder ,Touch the Sound" (GB 2004; R: T. Riedelsheimer; M: E. Glennie/F. Frith), das bewegende Portrait der weitgehend tauben schottischen Perkussionistin Evelyn Glennie, die mit über 100 Konzerten pro Jahr eine äußerst kreative und erfolgreiche Künstlerin ist.

Musik und bewegte Bilder gehen eine symbiotische Einheit ein, schon von Beginn an und nicht nur in dem Spezialfall „Musikfilm“. „Die beste Filmmusik ist die, welche man nicht selbständig hört ... Die beste Filmmusik ist die, welche mit der Filmszene un trennbar zusammenfließt" (Becce u. a. 1927, S. 45). Die Aussage wurde im Laufe der Film- geschichte öfter wiederholt und etliche Male filmmusikalisch belegt. Nicht erst das Mundharmonika-Motiv aus „Once upon a Time in the West" und imperiale Marschmotive aus Star Wars rufen imaginierte Kinobilder im Kopf ab.

Die lange Geschichte der Filmmusik erstreckt sich von den Pianobegleitungen der ersten cineastischen Versuche der Brüder Lumière 1895 in Paris bis zu den aufwändigen Hollywood-Blockbustern unserer Tage, die u. a. mit „Oskar“ ausgezeichneten Filmmusiken von Hans Zimmer und John Williams verknüpft sind. Eine aus gutem Grund angenommene symbiotische Beziehung von Film und Musik wirft viele Fragen auf. Wenn z. B. in der Begründung der Vergabe des deutschen Grimme-Preises 2005 an Klaus Doldinger zu lesen ist, die Musik befinde sich auf dem Weg zum ,integralen Bestandteil des audio-visuellen Gesamtkunstwerks Fernsehen", scheinen hier allerdings noch mehr Fragen aufgeworfen als tatsächlich Begründungen gegeben zu werden.

\section{Filmmusik im musikwissenschaft- lichen Diskurs}

Die genannten kurzen Schlaglichter verweisen auf die ca. 110-jährige Geschichte des Kinofilms, die an die ebenso lange und vielfältige Geschichte der Filmmusik gekoppelt ist. Kinofilme sind das traditionell bevorzugte Sujet filmmusikalischer Untersuchungen. Demgegenüber sind Analysen von Musik im täglichen filmischen Fernsehangebot unterrepräsentiert. Hier besteht ein Forschungsdefizit, beispielsweise in Bezug auf die musika-

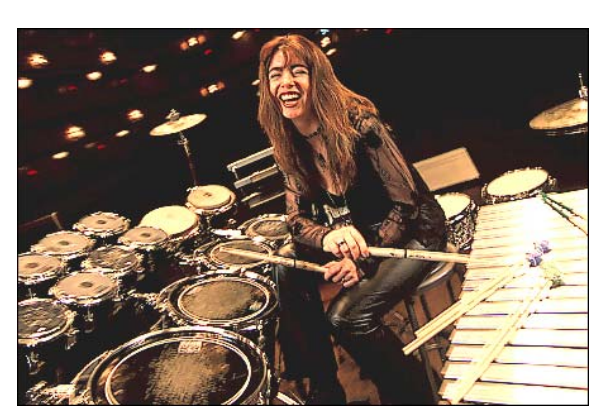

Evelyn Glennie

(Quelle: www.suchmanphoto.com/ portrait08.jpg) lisch-dramaturgische Ausgestaltung von Daily Soaps oder was die Wirkung von ständig präsenten Jingles oder musikalischen Logos betrifft, die Berührungspunkte zum Funktionskomplex Werbung aufweisen.

Filmmusik lässt sich wie jede andere Musik nach ästhetischen Kriterien analysieren. Umfassend und angemessen erschließt sich ihr Sinn je- 
doch erst im Beziehungsreichtum von Musik und bewegtem Bild. In dieser Hinsicht handelt es sich um funktionale Musik par exellence. Die Entwicklungsgeschichte vom Stummfilm zum Tonfilm und dann von analoger zu digitaler Produktion ist von technologisch tief greifendem Wandel begleitet. Denn ein nach Cue-sheets live spielender/improvisierender Kinoorganist der Stummfilmära, ein Komponist neoromantischer symphonischer Filmmusik und ein heutiger Sounddesigner repräsentieren jeweils sowohl unterschiedliche technischhandwerkliche Möglichkeiten, Kenntnisse und Zugriffsweisen, als auch differierende ästhetische filmmusikalische Konzepte, die sich in Relation zum jeweiligen funktionalen Kontext beschreiben lassen.

In dieser Hinsicht nimmt im musikwissenschaftlichen Diskurs die Frage nach der Funktionalität von Musik im (KinoFilm neben historischen bzw.

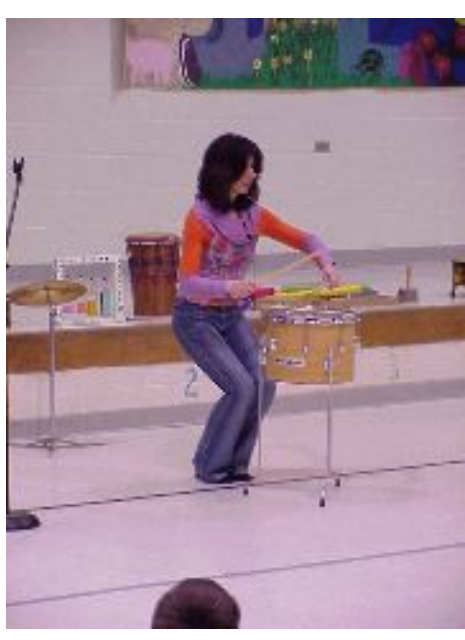

Evelyn Glennie (Quelle: www.forestview.dpsnc.net/student/ glennie/glennie.html) gogische Argumente für ihn. Besonders als Einstieg ist er praktikabel und besitzt das Potenzial zu interner Differenzierung. Beispiele von komplexeren Funktionssystematiken liefern Lissa 1965, Prendergast 1977, Emons/la Motte-Haber 1980, Schneider 1986, Maas 1994 und Bullerjahn 2001.

Filmmusik deckt ein weites Feld kompositorischer Praxis ab. Auch gegenwärtige Komponisten wie der oben genannte Hans Zimmer greifen auf historisch bewährte Techniken zurück, wenn es darum geht, Musik und Film in einen beabsichtigten Wirkungskontext zu stellen. Myriam Mayer (Mayer 2004) hat auf der Grundlage des exemplarischen Vergleichs verschiedener Spielfilmszenen aus unterschiedlichen Genres stilistischfunktionale Analysen erstellt, die es erlauben, eine Klassifikation wesentlicher filmmusikalischer Techniken Hans Zimmers vorzunehmen. Danach sind

niken Hans Zimmers vorzunehmen. Danach sind LeitmotivTechnik (auch Soundmotive, Erkennungszeichen), Mood-Technik (häufig in psychologisierender, ausdeutender Funktion) und kompilierende oder stilimitierende Verfahren zentrale Gestaltungsmittel seiner Filmmusiken. Diese Techniken bilden im Verbund eines hitverdächtigen Main-Titles die Grundlage für einen Soundtrack, der über die deskriptive und nachzeichnende Rolle von Filmmusik hinausgeht. In dem mit musikalischen Gesten und Vokabeln durchsetzten Stil- und Soundmix macht sich Zimmer Techniken zu Nutze, die schon in der Stummfilmzeit begründet und in Max Steiners Hollywood-Sinfonik intensiv zum Einsatz gekommen sind.

Mit der Filmmusik als einer Geschichte funktionaler Kontexte und kompositorisch-ästhetischer Techniken korrespondiert die Geschichte ihrer eigenen Rezeption, Interpretation und diskurshaften Erörterung, die sich teilweise in den o. g. Arbeiten widerspiegeln. Dabei ist nicht zutreffend, dass die Klassifikationssysteme, die am stärksten differenzieren, in jedem Fall den größten Erkenntnisgewinn nach sich ziehen. Vielmehr ermöglichen die unterschiedlichen funktionalen Ansätze filmmusikalische Beobachtungen aus bestimmten Perspektiven, indem ein jeweils spezifisches begriffliches Instrumentarium zur Anwendung kommt. So gesehen impliziert jeder Funktionskatalog bereits einen Interpretationsprozess, der die durch das Analyse-Instrumentarium ausgerichtete Wahrnehmung des Nutzers in spezifischer Weise filtert. Diese Tatsache hat 
pädagogische Konsequenzen, die im Folgenden entfaltet werden.

\section{Didaktische Verortung von Filmmu- sik als Thema des Musikunterrichts}

Die Themen von didaktischen Veröffentlichungen zur Filmmusik korrespondieren häufig mit der oben angeführten historisch-funktionalen Ausrichtung des musikwissenschaftlichen Filmmusik-Diskurses. „Filmmusik - Geschichte und Funktionen“ und „Filmmusik - in der Schule gemacht" lauten beispielsweise die entsprechenden Kapitel in der aktuellen Ausgabe des verbreiteten Lernwerks „Spielpläne 9/10“ aus dem KlettVerlag. Die Titel könnten repräsentativ für die didaktische Behandlung von Filmmusik im schulischen Unterricht stehen, sie stellen neben „Musik und Internet" oder „Videoclips" einen weiteren konkretisierten Teilaspekt des Komplexes „Musik und Medien“

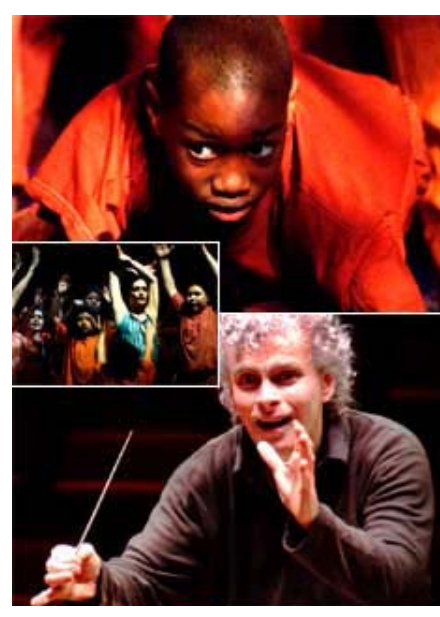

Rhythm is it

(Quelle: www.cine-plus.de/ production/ coproduktionen/ sir_simon.shtml) dar und sind in der fortgeschrittenen Sekundarstufe I zu verorten. Die Erarbeitung von historischen filmmusikalischen Entwicklungen und die exemplarische filmmusikalische Analyse auf der Grundlage von Funktionskatalogen bilden bislang Kerninhalte der Unterrichtsthematik.

Neben hier assoziierten gesprächsorientierten Methoden der Vermittlung sind praktische Schüler-Experimente zur filmmusikalischen Wirkung eine feste Konstante im Unterrichtsrepertoire, spätestens seit der umfassenden Etablierung des Gegenstands zu Beginn der 1980er Jahre. „Eine Szene - verschiedene Musiken“ gilt als ein exemplarisches Schüler-Experiment zum Thema Wirkung von Filmmusik (vgl. z. B. Maas 2001, S. 38). Die Erfahrung unterschiedlicher Wirkungen ein und derselben Filmszene durch Vertonung mit im Charakter unterschiedlichen Musikstücken verweist sowohl auf eine bestimmte Funktionalität (hier die Bedeutung verstärkende, eventuell Bedeutung stiftende (Quelle: http://www.stern.de/unterhaltung/film/ Funktion von Filmmusik) als 529814.html?nv=cp_L1_aa auch auf den Zusammenhang von Wirkung, Funktion und Interpretation im Wahrnehmungsprozess. Jede Entscheidung für ein System funktionaler Klassifizierung richtet die Aufmerksamkeit, d. h. die Wahrnehmung des Rezipienten, in spezifischer Art und Weise und steckt damit bereits ein Feld möglicher Bedeutungen bzw. Interpretationen ab. Musik bewirkt Aufmerksamkeitsfokussierung und je nach funktionaler Differenzierung wird so ein

mehr oder weniger offener Interpretationsrahmen nahe gelegt. Dabei ist Musik nicht beschränkt auf bloße Ergänzung der Bild- und Handlungsfolge, ist nicht nur plattes „Mickeymousing“, sondern kann zum Bedeutung generierenden Medium werden.

Auch der Wahrnehmungsprozess selbst impliziert ein kontingentes Feld der Bedeutungszuweisung. Der Wirkungszusammenhang von Auge und Ohr wurde früh erkannt und oft als konkurrierendes Verhältnis beschrieben (Arnheim 1932/2002, S. 304f., Kracauer 1964, S. 210, Behne 1987 und Schneider 1997). Rösing (2003, S. 10ff.) stellt dem neurophysiologische und rezeptionspsychologische Forschungsergebnisse gegenüber, die eher in Richtung eines permanent komplementären Zusammenwirkens der Sinne weisen. Erkenntnisse zu einer Konvergenz der Sinne (zsf. Marks 1978, S. 5ff.) reichen zurück bis in die Anfänge der Synästhesieforschung im 19. Jahrhundert. Wahrnehmung scheint demnach nicht eine Sache isolierter Sinnesorgane, sondern eines intermodalen Zusammenwirkens verschiedener Sinne zu sein. Rezeptionssituationen, auch die des Kinobesuchs, beziehen sich somit auf sinnlich mehrdimensionale Wahrnehmungsinhalte, wobei neben Intermodalität Informationsreduktion eine weitere Bedeutung generierende Komponente ist. Für das Entstehen und das Verarbeiten von Wahrnehmungsinhalten sind Eigenschaften der neuronalen Reizstrukturen verantwortlich, die durch Modalität (optisch, akustisch, haptisch etc.), Qualität (u. a. Klangspektrum), Intensität (z. B. laut - leise, stark -

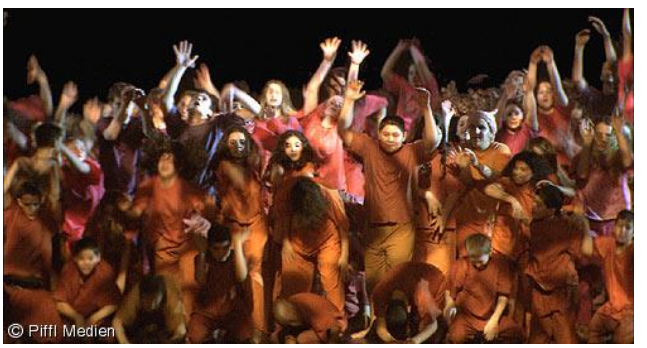
schwach), Zeitstruktur (Zeitdauer) und Ort (Lokalisation) beschrieben werden. Ohne hier auf die Grundlagen einer Theorie audiovisueller Wahrnehmung (Rösing 2003, S. 13ff.) zu rekurrieren, ist in diesem Zusammenhang von Bedeutung, dass die große Informationsdichte sinnlicher Eindrücke eine Informationsreduktion auf verschiedenen Ebenen neuronaler Verarbeitung erzwingt. Das führt dazu, dass nur bestimmte Ausschnitte, Ebenen aus dem gesamten audiovisuellen Informationsangebot bewusst rezipiert werden und zu bestimmten, subjektiv oder intersubjektiv relevanten Bedeutungskonstruktionen führen (vgl. dazu ausführlicher Rösing 2000, S. 15ff.). 
Didaktisch lässt sich aus diesen Erkenntnissen die Notwendigkeit ableiten, die Unterrichtsthematik „Musik und Film“ nicht nur ästhetisch und funktional, sondern stärker wahrnehmungsorientiert anzulegen, wobei die der Natur nach selektiv und intermodal angelegte Wahrnehmung intersubjektiv zu einer vielschichtigen ,interpretatorischen Unschärferelation“ (Rösing 2003, S. 22) führt, aus der methodisch und didaktisch Kapital zu schlagen sein dürfte.

\section{Didaktik der Film- musik im Kontext von Medien- und Filmbildung}

Eine Konsequenz aus einer stärker wahrnehmungsorientierten Didaktik „Musik und Film“ betrifft weniger das Film-

Verstehen mit Hilfe von Funktionskatalogen als das In-Gang-Setzen von Verständigungsprozessen, die der großen Bedeutung audiovisueller Medien für Kinder und Jugendliche Rechnung tragen. Filmmusik-Didaktik ist meistens auf Kinofilme bezogen. Feststellbar ist, und das entspricht wohl der vergleichbaren Situation in der Filmpädagogik, eine gewichtige Tendenz zu filmmusikalischer $\mathrm{Ka}$ nonbildung, die von Klassikern der Stummfilmära über Hollywoodsinfonik bis zu ambitionierten deutschen Produktionen reicht. Dabei ist die ästhetische Sensibilisierung mit filmischen Angeboten die eine Seite, die jugendliche Nutzung von Film als Identifikationsund Ausdrucksmittel mit spezifischen ästhetischen und kommunikativen Möglichkeiten die andere.

Vor diesem Hintergrund ist eine Stärkung von didaktischen Tendenzen wünschenswert, die „Musik und Film“ als Feld (musik)praktischen Handelns erschließen möchten. In didaktischen Reihen $\mathrm{zu}$ audiovisuellen Eigenproduktionen von Schülerinnen und Schülern konnte durchgängig festgestellt werden, dass es zu kurz greift, z. B. eigenproduzierte Musikvideos lediglich als Ausdruck von Aneignungsprozessen medialer Angebote zu verstehen (Imort 2002 und 2003). Demgegenüber erweist sich die Inszenierung des audiovisuellen Mediums als ein Ort der Verknüpfung von medial verschlüs- selten ästhetisierten Formen mit eigenen kulturellen und sozialen Verweisen. Der ästhetische Kontext wird also auch genutzt, um sich sozial zu verorten, Identität zu proben, Weltsicht zu artikulieren und Selbstbilder zu präsentieren. In dieser Hinsicht sind die Eigenproduktionen das Ergebnis von Verständigungsprozessen, in die eigene lebensweltliche Spuren eingeschlossen sind.

Die Entwicklung audiovisueller Ausdrucksformen benötigt Lernarrangements, die geeignet sind, entsprechende Gestaltungs- und Kommunikationsräume zu öffnen. Hier spielen auch Medienkompetenzen und Praktiken eine Rolle, die nicht im Kontext von Schule, sondern in außerschulischen, alltäglichen Zusammenhängen erworben wurden. Schülerinnen und Schüler sollten bestärkt werden, diese außerschulisch erworbenen Kompetenzen im Unterricht einzubringen und Musik und Film verstärkt als Entwicklungsstätte eigenständiger musikalischer Ausdrucksformen zu entdecken. Mögliche Impulse wirken hier aus medienpädagogischen Bereichen, die Film und aktive Medienarbeit verbinden. Unter musikpädagogischer Perspektive erscheinen daher Forschungen bzw. Konzepte wie VideoCulture (Niesyto 2003) und Chicam (Maurer 2004) besonders interessant. Eine verstärkte Zusammenarbeit von schulischer und außerschulischer produktionsorientierter Pädagogik ist also wünschenswert, wenn es um die Entwicklung von didaktischen Konzepten und Modellen geht, die Musik und Film als kreatives und künstlerisches Ausdrucksmedium fördern.

Die aktuellen BadenWürttembergischen Bildungspläne 2004 eröffnen in dieser Hinsicht weitgehende Handlungsspielräume, allerdings ist es Sache der einzelnen Schule, diese zu entwickeln und zu nutzen. Auch im Fach Musik werden Standards in Form von Kompetenzen und Inhalten formuliert. Intendiert ist, wie in anderen Fächern auch, die Ablösung der Belehrung (das Abarbeiten von Stoffplänen) durch eine Anstiftung zum selbstständigen Erwerb von Fähigkeiten, Kenntnissen und Verhaltensdispositionen (von Hentig, Einführung Bildungspläne 2004, S. 16). Der in Musik etablierte Unterrichtsgegenstand „Filmmusik“" wird unter dieser Prämisse nicht mehr 
explizit erwähnt, doch bietet der Rahmen, der durch Inhalte und Kompetenzen vorgegeben wird, einigen Spielraum für handlungsorientierte, musikpraktische Arbeit mit Medien. Informationstechnische Grundbildung ist nun fester Bestandteil aller Schulformen in den Sekundarstufen, sie zielt ausdrücklich nicht auf eine bloBe Handhabung der Technik, sondern auf einen kreativen, kritischen Gebrauch der neuen elektronischen Medien als Informations-, Kommunikations- und Ausdrucksmittel. Informationstechnische Grundbildung leistet damit einen wesentlichen Beitrag zur Medienkompetenz, darunter fällt Mediennutzung, Medienverständnis, Medienkritik und Mediengestaltung (vgl. z. B. Bildungsplan 2004 RS, S. 192). Im Gymnasium sind die Künste einer von sieben Kompetenzbereichen, in denen es um Auseinandersetzung mit ästhetischen Ausdrucksformen der Kultur durch Wahrnehmung, Reflexion sowie durch eigenes Gestalten und Darstellen geht (Bildungsplan Gymnasium 2004, S. 142). Herausgestellt werden fächerverbindende Arbeitsfelder wie Multimedia-Projekte. Künstlerischästhetische Ausdrucksformen spielen sowohl in bildender Kunst als in Musik eine bedeutende Rolle und können nicht einem Fach allein zugeordnet werden (S. 268). Vielleicht liegt es mit an der medienkritischen Einschätzung der Autoren („Die ständig wachsende Bedeutung medial vermittelter musikalischer Erfahrung reduziert die selbstbestimmten Eigentätigkeiten, die eigentlichen Primärerfahrungen mit Musik nehmen ab", S. 271), dass Musik und Medien kein Thema der informationstechnischen Grundbildung ist. Dem Musikunterricht der Realschule werden vielfältige Möglichkeiten zugestanden, moderne technische Medien im Unterricht einzusetzen (Computer, Studiotechnik, Videotechnik). Innerhalb der unterrichtlichen Handlungsfelder: Musik machen, Musik umsetzen, Musik hören, sich über Musik verständigen finden sich in den Kompetenzkonkretisierungen „die Wirkung von Musik erkennen“ (Jg. 8), „Bezüge zu anderen Künsten und Fächern herstellen" (Jg. 10) und „Computereinsatz“ (Jg. 6 bzw. 10). Im Bildungsplan Hauptschule erscheint Musik im Fächerverbund „Musik, Sport, Gestalten“. Lernen im Fächerverbund beruht auf dem Zusammenspiel von Wahrnehmung, eigenem Ausdruck und Nachdenken über den Prozess und das erreichte Ergebnis. Unterstützt werden soll die Persönlichkeitsbildung durch Förderung des Wahrnehmungs-, Vorstellungs- und Ausdrucksvermögens (Bildungsplan HS 2004, S. 142). Für unseren Zusammenhang relevante Inhalte sind „Tonträger und digitale Bilder“, es geht darum, „ästhetische Ausdrucksformen (zu) erproben, gestalten und genießen“ (S. 144).

Mit den Bildungsplänen 2004 ist der Rahmen für eine produktionsorientierte musikalische Arbeit mit Film gegeben, die Ausgestaltung und Etablierung obliegt der einzelnen Schule. Hier sind noch viele Schulcurricula zu konzipieren. Kollegien sollten, auch forschungsbegleitend, unterstützt werden, wenn es um die Konzeption und Entwicklung entsprechender Profilierungen geht.

\section{Literatur}

Arnheim, Rudolf (1932): Film als Kunst. München: Suhrkamp 2002.

Becce, Giuseppe / Erdmann, Hans / Brav, Ludwig: Allgemeines Handbuch der Filmmusik (2 Bde), Berlin / Leipzig: Lienau 1927.

Behne, Klaus-Ernst: Film - Musik - Video oder die Konkurrenz von Auge und Ohr. Regensburg: Bosse 1987.

Bildungspläne 2004. Hrsg. Ministerium für Kultus Jugend und Sport BW in Zusammenarbeit mit dem LEU Stuttgart 2004.

Bullerjahn, Claudia: Grundlagen der Wirkung von Filmmusik. Augsburg: Wißner 1999.

Emons, Hans / la Motte Haber, Helga de: Filmmusik. Eine systematische Beschreibung. München: Hanser 1980.

Imort, Peter: Audiovisuelle Eigenproduktionen von Schülerinnen und Schülern. Einige Ergebnisse und Perspektiven einer musikpädagogischen Projektreihe. In: Niesyto, Horst (Hrsg.): Selbstausdruck mit Medien. Eigenproduktionen mit Medien als Gegenstand der Kindheits- und Jugendforschung. München: Kopaed 2001, S. 141-156.

Imort, Peter: „Der Song sprach in Rätseln, so wie unser bisheriges Leben verlaufen war." Zur medialen Konstruktion musikalischer Lebenswelten in eigenproduzierten Musikvideos Jugendlicher. In: Jugend, Musik und Medien. Hrsg. von Renate Müller / Jens Heim / Patrick Glogner / Stefanie Rhein. Weinheim/München: Juventa 2002, S. $231-241$.

Kracauer, Siegfried (1963): Theorie des Films. Die Errettung der äußeren Wirklichkeit. Frankfurt/M.: Suhrkamp 1992.

Lissa, Zofia: Ästhetik der Filmmusik. Berlin: Henschel 1965.

Maas, Georg / Schudack, Achim: Musik und Film - Filmmusik. Informationen und Modelle für die Unterrichtspraxis. Mainz: Schott 1994.

Maas, Georg: Filmmusik. Arbeitsheft für den Musikunterricht in der Sekundarstufe 1. Leipzig/Stuttgart/Düsseldorf: Klett 2001.

Marks, Lawrence E.: The Unity of Senses: Interrelations among the Modalities. New York: Academic Press 1978.

Maurer, Björn: Medienarbeit mit Kindern aus Migrationskontexten. Grundlagen und Praxisbausteine. München: Kopaed 2004.

Mayer, Myriam: Der Filmkomponist Hans Zimmer - exemplarische Analysen neuerer Hollywood-Produktionen und ihre didaktische Um- 
setzung. Ludwigsburg (unveröff. Staatsexamens-Arb., Inst. Für Kunst Musik, Sport, PHL) 2004.

Niesyto, Horst (Hrsg.): VideoCulture. Video und interkulturelle Kommunikation. München: Kopaed 2003.

Pauli, Hansjörg: Filmmusik. In: Funkkolleg Musik, Studienbegleitbrief 11. hrsg. Vom Deutschen Institut für Fernstudien an der Univ. Tübingen. Weinheim und Basel / Mainz: Beltz und Schott 1978, S. 11-44.

Prendergast, Roy M.: Film Music - a neglected Art. New York: Columbia University Press 21992.

Rapée, Erno (1924): Motion picture moods for pianists and organists. New York: Arno Press, Nachdruck 1974.

Rösing, Helmut: Zur medialen Konstruktion musikalischer Lebenswelten. Eine kritische Bestandsaufnahme. In: Rösing, Helmut / Phleps, Thomas: Populäre Musik im kulturwissenschaftlichen Diskurs. Beiträge zur Popularmusikforschung 25/ 26. Karben: Coda 2000, S. 11-23.

Rösing, Helmut: Bilderwelt der Klänge Klangwelt der Bilder. Beobachtungen zur Konvergenz der Sinne. In: Clipped Differences. Geschlechterrepräsentationen im Musikvideo. Hrsg. von Dietrich Helms / Thomas Phleps. Bielefeld: transcript 2003, S. 9 - 25.

Schmidt, Hans-Christian: Filmmusik. Kassel: Bärenreiter 1982.

Schneider, Norbert Jürgen (1986): Handbuch Filmmusik I. Musikdramaturgie im neuen Deutschen Film. München: Ölschläger ${ }^{2} 1990$.

Schneider, Norbert Jürgen: Komponieren für Film und Fernsehen. Ein Handbuch. Mainz: Schott 1997.

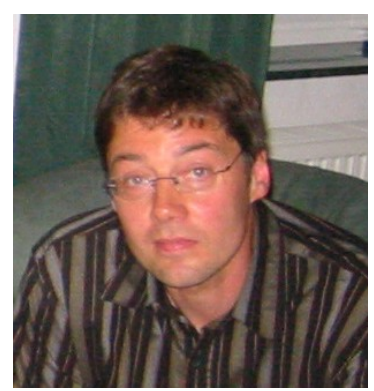

Prof. Dr. Peter Imort

Institut für Kunst, Musik und Sport an der Pädagogischen Hochschule Ludwigsburg, Abteilung Musik

Arbeitsschwerpunkte:

Kulturwissenschaftlich orientierte Musik- und Medienforschung, Digitale Musiktechnologien in der Populären Musik, Musikunterricht und Neue Medien, Forschungsprojekt „Musikvideo-Produktion in der Schule“. 